\title{
Présences du Cantique des Cantiques dans Le tombeau des rois d'Anne Hébert : incorporation et déplacements
}

\author{
ANNE-RENÉE CAILLÉ \\ UNIVERSITÉ DE MONTRÉAL
}

Résumé. S'il a déjà été établi que la Bible a enrichi et inspiré l'œuvre d'Anne Hébert, la présente étude s'attardera plus précisément aux présences du poème de l'Ancien Testament, le Cantique des Cantiques, au sein du recueil Le tombeau des rois. Nous verrons que les incorporations ponctuelles de différents motifs du texte biblique ne se font pas sans certains déplacements sémantiques qui suggèrent un rapport critique au texte sacré et surtout, à ses récupérations dogmatiques et morales. Au cœur de ce grand poème d'amour se trouvent les germes d'une union profanatrice que la poète prend le parti de faire sourdre, dans un acte de recréation exégétique inusité et lucide.

Mots-clés : Tombeau des rois, Cantique des Cantiques, Incorporation, Déplacement critique, Legs poétique, exégétique.

Il n'est plus original aujourd'hui d'affirmer les alliances entre l'œuvre d'Anne Hébert et la Bible. L'auteure confirme sans équivoque son influence en 1982 : «Cela fait partie de mon patrimoine, parce que la liturgie, les Écritures, I'Évangile concernaient les gens de ma génération. C'était non seulement des dogmes, une morale mais une culture aussi. C'était un enrichissement [...] C'est peut-être l'œuvre qui m'a marquée le plus... » (Vanasse, 1982 : 444) Bien que la dimension biblique de I'œuvre fut étudiée dans le passé ${ }^{1}$, la complexité du legs est telle qu'il est difficile de penser la question épuisée. L'attention sera ici précisément portée sur la cristallisation d'une filiation entre le Cantique des Cantiques et Le tombeau des rois. L'étude de la dissé-

1. Pensons aux recherches influentes d'Antoine Sirois : «Anne Hébert et la Bible », Voix et images, vol. 13, $n^{\circ} 3$ (39) : 459-472, 1988, et « Anne Hébert et la Bible. La suite... », Les Cahiers Anne Hébert, $n^{\circ} 11,2011$ : 115-138. Notons qu'Antoine Sirois a concentré ses recherches sur les récits et nouvelles de l'auteure. Adela Elena Gligor a pour sa part fourni une étude exhaustive des rapports de la Bible avec l'œuvre dans sa thèse de doctorat intitulée Mythes et intertextes bibliques dans l'œuvre d'Anne Hébert (Université d'Angers / Université de Montréal, 2008). 
mination fragmentaire du « plus grand des poèmes d'amour » dans le recueil mettra non seulement en parallèle les différentes manières de l'incarnation du legs, mais elle tentera de faire émerger la présence d'un mysticisme critique ${ }^{2}$. Si le Cantique est surtout célèbre pour son exposition d'un amour réciproque entre deux amants, qu'il soit d'ordre allégorique ou littéral, il faudra voir comment la poète en incorpore certains motifs tout en les déplaçant à des fins critiques. L'incorporation du texte canonique ne signifie pas pour autant que le texte source reste intouché; la poète ne propose pas un centon, mais procède d'un effort de recréation ${ }^{3}$ dont nous pèserons la teneur du grief. Par cette « réécriture » intermittente du Cantique dans Le tombeau des rois, la poète ne suggérerait-elle pas en filigrane une lecture exégétique? Ne se positionne-t-elle pas comme légatrice à son tour?

\section{Rapports préliminaires au canon}

Anne Hébert met en scène l'influence de la Bible dans son œuvre, entre autres par la citation textuelle, la reprise fragmentaire ou par un usage symétrique de symboles et métaphores récurrents. Dans l'extrait cité de l'entretien avec Vanasse, I'auteure parle de patrimoine et de culture. À l'évidence, son œuvre répond à son temps : la Bible devient un matériau d'enrichissement dans lequel puiser, et sa présence redouble la dimension fondatrice de son travail. Si I'on a reproché à son cousin SaintDenys Garneau un trop grand mysticisme, qui aurait presque avalé son œuvre ${ }^{4}$, il n'en est pas ainsi pour Hébert. Plusieurs indices portent à croire que la poète inscrit la parole biblique afin d'en exposer aussi la part d'ombre, celle-là même moralisatrice et dogmatique, pour reprendre sa propre terminologie, que la liturgie se plaît à mettre de l'avant à cette époque où le Verbe est récupéré dans une ritournelle vidée de signifiance, car figée dans une Vérité qui serait inaltérable, sorte de «psalmodie du chapelet ». (Hébert, 1960 : 9) Par sa nature de canon poétique, il n'est pas surprenant de voir apparaître le Cantique dans le Tombeau, que ce soit sous les traits de la figure de la femme-enfant, dans une lyrique amoureuse commune ou bien dans

2. Dans sa thèse, Adela Gligor s'intéresse aux « réécritures » de mythes et textes bibliques qui ont une portée critique dans un contexte historique, social et culturel québécois imprégné de l'idéologie cléricale. Ceci étant dit, précisons que l'auteure n'étudie pas la présence textuelle du Cantique des Cantiques dans I'œuvre poétique d'Anne Hébert et ne fait référence au texte biblique que pour souligner son influence globale sur la dimension « rythmique » de la poétique hébertienne.

3. J'emprunte l'expression à Gérard Genette, parlant du pastiche : « [Ce] n'est pas un centon, il doit procéder d'un effort d'imitation, c'est-à-dire de recréation. » (Genette, $1982: 85$ )

4. «Les silences prolongés du jeune homme, sa mystique de l'écriture, son aspiration cruelle vers la sainteté, tout consacrait en lui le stéréotype de celui pour qui la solitude était devenue [...] une condition d'échec supérieur, une maîtresse palpable. » (Bouchard, 1977 : 168) 
l'adresse à l'« amant ». Le Cantique, malgré la vastitude des exégèses, conserve son aura de mystère, et l'inscription poétique que propose Hébert contribue à la prolonger.

\section{Le Poème des Poèmes}

Le Cantique demeure un des textes bibliques les plus interprétés, cités et pastichés. Sa richesse herméneutique semble sans mesure, et sa nature poétique participe certainement à sa polysémie, tout comme l'intrication des voix et adresses complexifie I'identification énonciative. Selon les époques, les traditions et les différentes traductions, les lectures du texte sont appelées à se modifier. Il ne s'agira pas ici d'enquêter afin de savoir quelles sources ou traductions exactes furent utilisées par Hébert, dans la mesure où la reprise textuelle peut aussi se faire de mémoire ou par des réminiscences plus inconscientes. Il sera plus pertinent de se demander comment l'imaginaire du Cantique apparaît, quelles topiques sont privilégiées par la poète, quels déplacements sont opérés et quelles sont leurs fonctions dans l'économie du recueil.

Les différents sens octroyés au poème (marial, marital, érotique, spirituel...) se divisent souvent entre deux vastes catégories d'approches : littérale ou allégorique ${ }^{5}$, le Cantique étant le plus souvent interprété soit comme poème charnel ou figuration de l'union entre le Seigneur et son peuple (ou l'Église), soit comme illustration d'une quête mystique (Millet-Gérard, 2010 : 68). Il sera démontré que la récupération d'Hébert semble a priori se situer du côté des interprétations conjugales « modernisantes », mais nous verrons que l'œuvre réussit à échapper à ce binarisme.

Renversements des codes amoureux : le désir

« Certains exégètes estiment que l'auteur principal du livre est une femme. Même si I'on n'adopte pas cette thèse, on doit reconnaître que c'est I'un des textes bibliques qui accordent à la voix féminine une place de premier plan. » (La Bible [nouvelle traduction], 2001 : 2900) La voix féminine occupe assurément une position excep- 
tionnelle dans I'Ancien Testament, et son affirmation est inhabituelle ${ }^{6}$. Au sein du dialogue entre l'amant et l'amante (et les autres personnages), il est aussi à noter que les interventions de cette dernière sont plus nombreuses et qu'elle occupe davantage la parole que I'homme qu'elle aime ${ }^{7}$. Antoine Sirois observe certains processus de féminisation dans l'intertexte biblique d'Hébert (Sirois, 2011 : 129), et cela paraît éloquent dans le cas du Tombeau, dont le sujet poétique est féminin. Il est d'ailleurs décrit à l'image de l'amante du Cantique, jeune fille à la lisière de la maturité, entre deux âges. Alors que I'amante du Cantique accueille son désir avec naïveté, candeur et envie, celle du Tombeau semble déjà désillusionnée quant à l'effervescence du désir ou alors en lutte contre ce devenir-femme désirant qui n'évoque que la menace. Ce désir auquel on octroie une nature perverse et malfaisante est typique de l'œuvre hébertienne.

Jean-Jacques Lavoie observe que le mot désir (shûq) n'apparaît que deux fois dans la Bible, soit dans la Genèse et dans le Cantique, mais que les occurrences ont des connotations opposées : «Par la reprise de ce mot rare, Ct 711 est donc un véritable détournement de texte à transformation maximale. La malédiction de Gn 316 est changée en bénédiction. Le désir est une joie et non un jugement. La relation amoureuse n'est plus unilatérale ». (Lavoie, $1992: 55$ )

Gn 3, 16 : À la femme, il dit : « Je ferai qu'enceinte, tu sois dans de grandes souffrances; c'est péniblement que tu enfanteras des fils. Ton désir te poussera vers ton homme et lui te dominera. »

Ct 7, 11 : Je suis à mon bien-aimé, et vers moi se porte son désir8.

Dans le Cantique, le désir unit la femme à I'homme, il est partagé et réciproquement exprimé, comme en témoignent leurs éloges respectifs. La possession et l'appartenance opèrent dans les deux sens : il y a rencontre du désir, de l'amour et des corps. Ce n'est plus la femme qui est soumise à I'homme : tous deux deviennent

6. Julia Kristeva parle de cette « affirmation de la femme»: «Elle, l'épouse, prend pour la première fois au monde la parole devant son roi, époux ou Dieu; pour s'y soumettre soit. Mais en amoureuse aimée. C'est elle qui parle et qui s'égale, dans son amour légal, nommé, non coupable, à la souveraineté de l'autre. La Sulamite amoureuse est la première femme souveraine devant son aimé. Hymne à l'amour du couple, le judaïsme s'affirme comme une première libération des femmes ». « Une sainte folie : elle et lui », Histoires d'amour. Cité dans Anne Mars. Le Cantique des Cantiques, du roi Salomon à Umberto Eco : anthologie, Paris, Cerf, 2003 : 285.

7. Cette observation est rendue possible grâce aux choix éditoriaux de la Traduction œcuménique de la Bible (TOB), qui indique tous les différents allocutaires, contrairement à l'édition d'André Chouraqui.

8. Shûq est traduit aussi par « élan » $(T O B)$ ou « passion » (Chouraqui). La Bible de Jérusalem le traduit par « désir » dans Ct 7, 11. Pour la citation de Gn 3, 16 par contre, la traduction est « convoitise », alors j'ai privilégié la traduction de la $T O B$ pour le bien de la démonstration. 
soumis au désir qui les unit, et cette soumission est tout autre, car embrassée par les deux parties. Ces deux versets montrent bien la réciprocité du désir : « Mon chéri est à moi, et je suis à lui, qui paît parmi les lis » (Ct $\left.2,16^{\circ}\right)$ et « Je suis à mon chéri et vers moi est son élan. » $(C t 7,11)$ Ces versets ponctuent d'ailleurs le Cantique à la façon de refrains qui réactualisent cette réciprocité du désir.

Dans le Tombeau, le désir et l'union des amants ne sont pas des données positives. Les « époux » sont « [t]ristes [...] tranchés et perdus. » (« La chambre fermée », Fuvre poétique 1950-1990, $2000: 36$ ), et les amants sont étêtés : « Nous les filles bleues de l'été / [...] Dans nos mains peintes de sel / [...] Nous tenons d'étranges lourdes têtes d'amants / Qui ne sont plus à nous / Pèsent et meurent entre nos doigts innocents. » («L'envers du monde »10 : 45) L'amante étête son bien-aimé, dans un geste libérateur et disculpé. Constamment diabolisé, « [I]e désir rôde vole et poudre ». (《Retourne sur tes pas » : 41) La fusion des corps désirants louangée dans le Cantique est, dans le Tombeau, ramenée à la malédiction et à la soumission de la Genèse : la femme reste subordonnée à I'homme dont elle est issue ${ }^{11}$. Hébert insiste à plusieurs reprises sur cet asservissement, et l'atmosphère de cloisonnement constant dans lequel se trouve la femme en témoigne. Il serait juste de souligner qu'un désir menaçant « rôde » aussi, en filigrane, dans le Cantique. Hébert semble rappeler que le désir oppressif, initial, de la Genèse n'est jamais loin, même dans ce grand poème amoureux.

\section{Amante cloisonnée, amante martyre}

Dans le Tombeau, la poète prend le parti de dépeindre l'amante dans un état qui se veut postérieur au désir positif, naissant et éclatant décrit dans le Cantique. Elle décide de le miner. La figure féminine est placée à maintes reprises dans un état de lucidité presque violent. D'abord, I'heure du jour la plus souvent évoquée est midi

9. Sauf si indiqué, les extraits du Cantique des Cantiques sont tirés de la Traduction œcuménique de la Bible, 2004 : 1006-1014.

10. Par souci de clarté, j'indiquerai les titres des poèmes cités dans le corps du texte, poèmes qui proviennent, sauf si indiqué, du Tombeau des rois, de l'édition Fuvre poétique 1950-1990 : 7-54.

11. Gn 2, 22-25 : «Puis, de la côte qu'il avait tirée de I'homme, Yahvé Dieu façonna une femme et I'amena à I'homme. Alors celui-ci s'écria : "Pour le coup, c'est l'os de mes os et la chair de ma chair! Celle-ci sera appelée femme, car celle-ci fut tirée de l'homme, celle-ci." C'est pourquoi I'homme quitte son père et sa mère et s'attache à sa femme, et ils deviennent une seule chair. » (La Bible de Jérusalem, $2009: 23$ ). Notons qu'Hébert reprendra le syntagme « chair de ma chair » dans le poème «Amour » : « Toi, chair de ma chair, matin, midi, nuit, toutes mes heures et mes saisons ensemble ». («Poèmes anciens », Fuvre poétique 1950-1990: 106) 
$\left(O P^{12}: 16,38,50^{13}\right)$, qui est la seule heure présente littéralement dans le Cantique (Ct 1,7$)$. Selon une interprétation symbolique biblique, l'ensoleillement est à son point le plus ardent à midi et donc, I'heure représente un temps de vérité, un temps théophanique, un temps de révélation (Millet-Gérard : 176-178). Ce soleil puissant brûle les mains («Les pêcheurs d'eau »: 16); il noircit la peau : « Midi brûle auxcarreaux d'argent / La place du monde flambe comme une forge / L'angoisse mefait de l'ombre / Je suis nue et toute noire sous un arbre amer. » ( « La chambre de bois » : 38; je souligne) La figure féminine d'Hébert se décrit sous les mêmes traits que l'amante au début du Cantique : « Je suis noire, moi, mais jolie, filles de Jérusalem [...] Ne faites pas attention si je suis noiraude, si le soleil m'a basanée. Mes frères m'ont tannée : ils m'ont mise à surveiller les vignes; ma vigne à moi, je ne l'ai pas surveillée. » (Ct 1, 5-6; je souligne) Ce qui se révèle aux deux femmes dans la pleine lumière, leur « illumination intérieure » du midi (Millet-Gérard : 233), c'est qu'elles sont entrées dans le désir. Dans le Cantique, les frères ont laissé leur petite sœur surveiller les vignes, alors que leur sœur était inconsciente face à la surveillance de sa propre «vigne ». Ainsi, dans le même verset, le sens littéral de vigne est uni à son sens allégorique, relatif à la fécondité. L'amant sera pour sa part comparé, dans les versets suivants du Cantique, à un pommier : «À son ombre, selon mon désir, je m'assieds ». (Ct 2, 3) Hébert non seulement évoque une figure jumelle à la jeune femme noire du Cantique, mais elle ajoute aussi la même ombre qui la surplombe, à la différence près qu'elle la teinte de la malveillance d'un arbre dit « amer ».

Plusieurs métaphores qui décrivent l'amante « cloisonnée » du texte biblique sont reprises dans le Tombeau. La « colombe au creux d'un rocher » (Ct 2, 14) s'apparente à l'image récurrente de l'oiseau captif du Tombeau : «Les pêcheurs d'eau / Ont pris l'oiseau / Dans leurs filets mouillés » («Les pêcheurs d'eau » : 16) ou bien celui, mort, qui gît « [d]ans un bocage inconnu. » («La voix de l'oiseau » : 21) Hébert en renverse la teneur, car, dans le texte source, la colombe captive reste « cachée » par mesure de protection, alors que la poète ne fait qu'en conserver la nature d'emprisonnement.

Dans le Cantique, plusieurs métaphores de cloisonnement réfléchissent la virginité de l'aimée : «Tu es un jardin verrouillé, ma sœur, ô fiancée; une source verrouillée, une fontaine scellée! » (Ct 12; je souligne) Alors que le «jardin scellé » renverrait à une iconographie mariale (Millet-Gérard : 65), les symboles de la vigne, de la source,

12. OP pour Fuvre poétique 1950-1990, 2000.

13. Respectivement «Les pêcheurs d'eau », «La chambre de bois » et « Un bruit de soie ». 
de la fontaine ou du puits renvoient tous à la fécondité. Ce sont les frères et la mère de la jeune amante qui la «verrouillent » ou la «scellent». Le texte insiste sur ce cloisonnement en l'opposant au désir naissant de la jeune femme et à l'avancée discontinue de son amant : «Mon chéri avance la main par le trou; et mon ventre s'en émeut. [...] [M]es mains distillent de la myrrhe [...] sur les paumelles du verrou. » $(C t 5,4)$ La virginité de l'amante est ainsi préservée derrière les différents verrous, remparts ou murailles que la mère et les frères ont érigés devant leur fille et sœur contre la proie qui vient vers elle: «Saisissez-nous les renards, les petits renards qui ravagent les vignes, alors que notre vigne est en bouton! » (Ct 2, 15) L'intermittence de la présence de l'amant comme celle de la surveillance de l'amante performent une aptitude même au désir qui est ambivalente. Il est intéressant de voir que l'amant est décrit par la mère comme une bête qui pourrait détruire la « vigne » bourgeonnante. Ce dernier attribut laisse croire une fois de plus que la jeune fille serait « prête » pour I'amour. Pourtant les frères la décriront autrement : « Nous avons une sœur. Elle est petite: elle n'a pas de seins » $(\mathrm{Ct} 8,8)$, description qui soutient plutôt qu'elle est encore une enfant. Cela atteste que la figure de la jeune fille est tout juste à la lisière de la maturité et que le tiraillement entre les deux âges est persistant.

La figure féminine du Tombeau renvoie une fois de plus à la jeune sœur, comme en témoigne cette description corporelle : « Je suis une fille maigre / Et j'ai de beaux os. » («La fille maigre » : 29) Elle est tour à tour captive, enclose, emmurée, comme l'amante du Cantique, mais ce n'est pas, chez elle, dans le but de préserver sa virginité, au contraire. Cette dernière a déjà été prise, profanée, et la captivité en est le corollaire : «Un mur à peine / Un signe de mur / Posé en couronne / Autour de moi. / Je pourrais bouger / Sauter la haie de rosiers, / L'enlever comme une bague / Pressant mon cœur [...] Seule ma fidélité me lie / Ô liens durs / Que j'ai noués ». (« Un mur à peine » : 33) Soulignons les liens avec le texte biblique. D'abord, le mur est décrit comme une couronne, que l'on peut associer à celle qui incarne le mariage du roi (Ct 3,12), surtout que le poème d'Hébert insiste sur l'alliance du mariage («bague », « fidélité », « liens noués »). Évidemment, plusieurs autres poèmes appartiennent au registre thématique de l'enfermement («La chambre fermée », « La chambre de bois », « De plus en plus étroit», « Vie de château »). Dans I'optique de montrer les effets d'appropriation précis provenant du Cantique, certains extraits choisis montreront une symétrie. 
Le Cantique fait ainsi référence à la « couche » des amants : « Les poutres de notre maison sont les cèdres, et nos lambris, les genévriers. » (Ct 1,17$)$ Le Tombeau dessine une maison des amants qui est fort semblable, faite de «bois ancien », comparée à un coffre ou une armoire de bois à l'odeur de pin (« La chambre de bois », $O P$ : 37-38) qui encercle l'amante, jusqu'au télescopage même du « lit de bois noir [qui la] contient » («La chambre fermée »: 36) Dans les deux cas, le bois nommé provient de conifères. Cependant, le renversement négatif par rapport au Cantique est une fois de plus éloquent dans le passage de la « verdoyante couche » des amants à celle où l'amante est dépeinte en train de brûler, les « bras étendus » en forme de « croix tremblante » («La chambre fermée » : 35). La violence, chez Hébert, émerge de l'union; même si les deux époux sont «tranchés et perdus », la femme est celle qui est le plus souvent dans une position de martyre. Elle est non seulement oppressée mais démembrée et meurtrie : son cœur est pillé, ses prunelles, crevées, son odeur, capiteuse. Dans l'extrait cité, la position christique de l'épouse dénote à la fois la condamnation et le rachat d'une faute. Qu'expie-t-elle? Il est à se demander si la faute qui est sans arrêt réactualisée n'est pas celle-là même d'être une femme, telle que la Genèse I'entendait.

Si le Cantique est souvent reconnu pour sa représentation d'une réciprocité amoureuse qui se manifeste dans la joie et les éloges, I'homme est néanmoins perçu comme une menace par la mère (« renards »). De plus, les frères veulent protéger leur sœur contre une attaque ou un enlèvement : «Si elle était un rempart, nous bâtirions sur elle des créneaux d'argent. Si elle était une porte, nous la bloquerions d'une planche de cèdre. » $(\mathrm{Ct} 8,9)$ Nous avons vu qu'Hébert récupérait ces éléments et les accentuait. Il paraît essentiel de mettre en évidence que le Cantique comme la Genèse, quoique de façon moins impérative, sous-tend aussi que la femme représente une menace dont I'homme devrait se méfier ou qu'il devrait tenter d'asservir.

Hébert ne procède pas seulement par déplacements de motifs du Cantique, elle incorpore certains éléments en germe dans le texte (et qui sont moins souvent exposés) dans l'économie de ses exégèses. À deux reprises, l'amante est décrite comme «terrible», d'abord par l'amant et ensuite par les « Amoureuses » (le harem), qui le réitéreront : «Tu es belle, ma compagne, comme Tirça, jolie comme Jérusalem, terrible comme ces choses insignes », dira le bien-aimé. (Ct 6,4) Les deux autres traductions consultées proposent une teneur parente : « redoutable comme des bataillons » (La Bible de Jérusalem) et «terrible comme un mirage » (Chouraqui). Pourquoi une femme terrible ou redoutable émerge-t-elle brusquement dans le poème? 
Ne serait-ce pas parce qu'en faisant émerger le désir de l'homme, elle possède la capacité de lui ravir non seulement son cœur, mais possiblement sa raison : « Tu me rends fou, ma sœur, ô fiancée ». (Ct 4, 9) Cela est sans issue : I'homme aussi sera soudé à la femme : «[S]es mèches sont comme la pourpre : un roi est enchaîné par ces flots. » $(\mathrm{Ct} 7,6)$ Dans le Tombeau, les positions d'enchaînement sont inversées, dans ce qui semble être une sorte d'anticipation de sa «terreur enchaînante » : afin de la prévenir, le sujet féminin sera opprimé, enfermé et même préservé jusque dans la mort.

\section{L'amant-reliquaire, l'amante-relique}

Le va-et-vient des amants qui se perdent et se retrouvent établit la dynamique du poème biblique. En Ct 3, 4, l'amante regagne une fois de plus son amant perdu et affirme : « Je le saisis et ne le lâcherai pas [...] », ce qui n'est pas sans rappeler ces vers de « La fille maigre » : «Un jour je saisirai mon amant / Pour m'en faire un reliquaire d'argent. » $(O P$ : 39; je souligne) Les reprises lexicales de l'amant et de la saisie sont indéniables. Un même désir de possession durable s'énonce chez Hébert, bien que la temporalité ne soit pas la même : la possession est différée, aura lieu un jour, dans un futur indéterminé. Dans les deux cas, c'est la femme qui veut « saisir » I'homme, la mainmise étant donc renversée. Le vers second, « [P]our m'en faire un reliquaire d'argent », ne doit pas être isolé dans la reprise. Son ajout significatif est primordial dans la relation qui unit le poème source à celui du Tombeau.

Dans la structure du Cantique, le chant cinq est consacré à l'éloge de l'amant par l'amante, qui comparera tous les membres de son corps à des pierres ou des bijoux précieux : «Ses mains sont des bracelets d'or remplis de topazes. Son ventre est une plaque d'ivoire couverte de saphirs, ses jambes sont des piliers d'albâtre fondés sur des socles d'or fin. » (Ct 5, 14-15) Le corps de I'homme devient le réceptacle de toutes les richesses. Les métaphores intensifient bien entendu l'aura de désir qui se dégage de l'objet («tout son être est l'objet même du désir », dira-t-elle ensuite) et elles impliquent que la valeur du bien-aimé est décuplée aux yeux de la femme. Dans cette perspective, le poème d'Hébert préalablement cité attire d'autant plus l'attention, alors que l'amant est comparé à un reliquaire d'argent, c'est-à-dire un réceptacle (boîte ou coffret) où sont insérées ou fixées des reliques. Les deux amants se transforment ainsi en reliquaires dans lesquels les amantes-reliques habiteront à jamais. La saisie prend d'ailleurs un sens inhabituel, plus passif, de l'ordre d'une inscription en l'autre d'une alliance qui serait infinie. 
Ces vers du Cantique consolideront cette lecture : « [M]ets-moi comme un sceau sur ton cœur, comme un sceau sur ton bras. Car : Fort comme la Mort est Amour », dira l'amante à son amant en Ct 8,6. Cette adresse permet de confirmer I'hypothèse de l'amant-reliquaire et de l'amante-relique. Le sceau signifie non seulement qu'elle s'inscrit sur le corps de I'homme, mais qu'elle signe la pérennité de leur alliance de façon visible. La Traduction œcuménique de la Bible ajoute dans son appareil critique que le «sceau pouvait se porter en pendentif autour du cou ». (2004: 1013, Ct $8,6 p$ ) Hébert se réappropriera cette signification dans « La fille maigre » : « Un jour je saisirai mon amant / Pour m'en faire un reliquaire d'argent. / Je me pendrai / À la place de son cœur absent. / [...] Et parfois / En [s]a poitrine, fixée, / J'entr'ouve / Mes prunelles liquides ». (OP : 29-30; je souligne) Une fois de plus, I'assimilation des motifs du texte biblique est modifiée par la poète : I'amante du Tombeau, qui se pend tel un sceau au cœur de son amant, se transforme en amante pendue, sa mort enclose dans la poitrine de son bien-aimé, qui la portera à jamais. Les deux amants deviennent les dépositaires de leurs amantes ; dans le Cantique, c'est l'alliance amoureuse qui se doit de survivre à la mort dans le corps de l'amant, alors que dans le Tombeau, on insiste sur une alliance qui est scellée par la mort (ou par la morte) même qui y restera à tout jamais « enclose ». Cette dernière lecture est d'autant plus pertinente lorsqu'on pense la relique comme un « reste [...] des martyrs » (Littré, 2004 : 1187), alors qu'il a été démontré plus tôt que la femme hébertienne possédait certains traits d'une martyre.

Le psychanalyste Pierre Fédida, dont certains travaux portent sur le deuil, établira des rapports entre la perte d'un proche et la relique : « [L]a relique prend sens dans le désir de conserver quelque chose de ce dont on se sépare sans pour autant, devoir renoncer à s'en séparer. » (Fédida, 1978 : 76; je souligne) Dans le Cantique, on insistera pour dire que l'amour est plus fort que la mort. Le poème d'Hébert laissera plutôt planer la survivance de l'amante morte dans le corps de son amant, qui sous-entend davantage un désir de hantise chez l'absente que celui de faire durer le souvenir d'un amour « romantique » éternel. Il reste que dans les deux textes, la figure de la relique suppose un processus de conservation qui ferait suite à la séparation et qui permettrait de garder intact et vivant quelque chose de l'union, que cela soit un désir partagé par les deux parties ou imposé par une amante déçue. Notons que dans « La fille maigre », l'amante se veut entièrement introduite à l'intérieur de l'amant, qui n'est plus strictement métamorphosé en « reliquaire », mais dès lors en tombeau. L'amante se fantasme comme à jamais conservée en son amant. Il s'agit 
d'un fantasme de mémoire, d'union et de saisie qui défie toute possibilité de perte, de rejet ou d'oubli et qui doit aussi être compris comme un fantasme d'incorporation inversée.

\section{Incorporation mélancolique et critique du texte biblique}

Les modifications opérées à même la récupération des motifs du Cantique portent à croire qu'Anne Hébert ne tentait pas simplement de commémorer le plus grand poème d'amour dans ce qui aurait pu être un acte strictement mélancolique. L'acte d'incorporation est aussi un acte de jugement.

L'incorporation devrait peut-être être envisagée dans la perspective du concept psychanalytique, qui est lié au deuil et, plus précisément, à la mélancolie face à la perte d'un objet. Le deuil s'accomplit lorsque «l'épreuve de réalité a montré que l'objet aimé n'existe plus et édicte l'exigence de retirer toute la libido des liens qui la retiennent à cet objet. » (Freud, 1968 : 148) Vraisemblablement, Hébert témoigne de la présence fondamentale du texte biblique au sein de son œuvre et en souligne la force d'enrichissement. Ceci étant dit, les modulations appliquées au texte laissent aussi croire que cette réactualisation n'est pas seulement de l'ordre de l'enrichissement. Dans l'étude qui nous intéresse, il semble que la poète ne veuille pas laisser aller l'objet biblique et que, par le processus d'incorporation, elle le fasse pénétrer dans le corps de son texte. Sa voix devient le canal qui mène au support de conservation, le poème. Cependant, le rapport avec l'objet « perdu » n'en est pas un de fusion, mais de grande ambivalence. Les motifs positifs des louanges amoureuses sont transformés en affects négatifs, et les assimilations du Cantique insistent sur la position de soumission et d'oppression de la figure féminine, un parti pris exégétique inhabituel. Il semble juste de croire qu'Hébert garde certains aspects du Cantique intacts en se les réappropriant tout en en malmenant une récupération dogmatique qui ferait de l'histoire charnelle, le sens littéral, une représentation de la fidélité et encore une fois, intriquée en elle, celle de la soumission de la femme à son bien-aimé. Hébert écrira d'ailleurs, quelques années après le Tombeau, dans le poème «La ville tuée » : «[L]e recours à Dieu périmé [...] / Les enfants furent endormis de force sans bruit. On érigea le dogme et la morale, et la première saison s'allongea sans passion ». ( $O P: 83)$ C'est contre cela qu'Hébert révèle l'origine, sournoise, d'une morale pernicieuse présente entre les lignes du Cantique. Par sa recréation intermittente du texte, elle fait intervenir une critique de la liturgie et de la morale ambiante du Québec des années cinquante. Loin de vouloir rejeter ni le texte biblique 
ni la religion catholique, elle s'associe à quelques reprises à la revue française Esprit, qui manifeste la volonté d'envisager le catholicisme hors des frontières du dogme et de la pensée réactionnaire et d'opter pour une perspective progressiste. Cela semble être tout à fait emblématique du legs critique que choisit d'accomplir Anne Hébert dans certains poèmes du Tombeau des rois avec son incorporation et ses déplacements de fragments du Cantique des Cantiques. Obliquement, elle suggère que la transmission d'un modèle textuel est loin d'être un processus unilatéral et qu'elle ne devrait jamais être un acte immuable. 


\section{Bibliographie}

[S.n.] (2004), Dictionnaire Nouveau Littré, Paris, Garnier.

[S.n.] (2004), Traduction œcuménique de la Bible, trad. des textes originaux hébreu et grec, [Paris], Société biblique française et Éditions du Cerf.

[S.n.] (1989), La Bible, trad. et présentée par André Chouraqui, Paris, Desclée de Brouwer.

[S.n.] (2009), La Bible de Jérusalem, trad. sous la direction de l'École biblique de Jérusalem, Paris, Cerf.

BOUCHARD, Denis (1977), «Anne Hébert et la "solitude rompue" : tentative de démythification d'un des lieux communs de notre littérature », Études françaises, vol. 13, n 1-2 : 163-179.

BOYER, Frédéric (dir.) (2001), La Bible, Paris, Bayard.

ENA, Jean Emmanuel de (2004), Sens et interprétations du Cantique des Cantiques, Paris, Cerf.

FÉDIDA, Pierre (1978), "La relique et le travail du deuil », dans L'absence, Paris, Gallimard, coll. «Connaissance de l'inconscient » : 75-84.

FREUD, Sigmund (1968), « Deuil et mélancolie », dans Métapsychologie, trad. par J. Laplanche et J.-B. Pontalis, Paris, Gallimard : 147-174.

GENETTE, Gérard (1982), Palimpsestes : La littérature au second degré, Paris, Seuil.

GLIGOR, Adela Elena (2008), Mythes et intertextes bibliques dans l'œuvre d'Anne Hébert, thèse de doctorat, Université d'Anvers / Université de Montréal.

HÉBERT, Anne (1960), « Quand il est question de nommer la vie tout court, nous ne pouvons que balbutier », Le Devoir, 22 octobre : 9 et 12.

HÉBERT, Anne (2000), CEuvre poétique 1950-1990, Montréal, Boréal.

LAVOIE, Jean-Jacques (1992), « Jeux bibliques d'intertextualité ou l'impossibilité de lire hors de la bibliothèque », Tangence, $\mathrm{n}^{\circ} 35: 46-58$.

MARS, Anne (2003), Le Cantique des Cantiques, du roi Salomon à Umberto Eco : anthologie, Paris, Cerf. MILLET-GÉRARD, Dominique (2010), Le signe et le sceau. Variations littéraires sur le Cantique des Cantiques, Genève, Droz.

SIROIS, Antoine (1988), « Bible et récits d’Anne Hébert », Voix et images, vol. 13, n³ (39) : 459-472.

SIROIS, Antoine (2011), « Anne Hébert et la Bible. La suite... », Les Cahiers Anne Hébert, $n^{\circ} 11$ : 115-137.

VANASSE, André (1982), "L'écriture et l'ambivalence, entrevue avec Anne Hébert », Voix et images, vol. $7, \mathrm{n}^{\circ} 3: 441-448$. 